\title{
Raman-Activated Droplet Sorting (RADS) for Label-Free High- Throughput Screening of Microalgal Single-Cells
}

\author{
Xixian Wang, ${ }^{\dagger, \S, \perp \odot ~ L i h u i ~ R e n, ~}{ }^{\dagger, \S, \perp}$ Yetian Su, ${ }^{\dagger}$ Yuetong Ji, ${ }^{\dagger, \S}$ Yaoping Liu, ${ }^{\|}$Chunyu Li, ${ }^{\dagger, \S}$ Xunrong Li, ${ }^{\dagger, \S}$

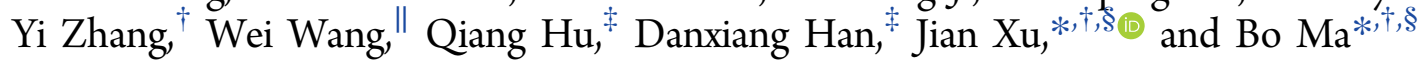 \\ ${ }^{\dagger}$ Single-Cell Center, CAS Key Laboratory of Biofuels and Shandong Key Laboratory of Energy Genetics, Qingdao Institute of \\ Bioenergy and Bioprocess Technology, Chinese Academy of Sciences, Qingdao, Shandong 266101, China \\ ${ }^{\ddagger}$ Center for Microalgal Biofuels and Biotechnology, Institute of Hydrobiology, Chinese Academy of Sciences, Wuhan, Hubei 430072, \\ China \\ ${ }^{\S}$ University of Chinese Academy of Sciences, Beijing 100049, China \\ "National Key Laboratory of Science and Technology on Micro/Nano Fabrication, Institute of Microelectronics, Peking University, \\ Beijing 100871, China
}

\section{Supporting Information}

ABSTRACT: Raman-activated cell sorting (RACS) has attracted increasing interest, yet throughput remains one major factor limiting its broader application. Here we present an integrated Raman-activated droplet sorting (RADS) microfluidic system for functional screening of live cells in a label-free and high-throughput manner, by employing AXT-synthetic industrial microalga Haematococcus pluvialis (H. pluvialis) as a model. Raman microspectroscopy analysis of individual cells is carried out prior to their microdroplet encapsulation, which is then directly coupled to DEP-based droplet sorting. To validate the system, H. pluvialis cells containing different levels of AXT were mixed and underwent RADS. Those AXT-hyperproducing cells were sorted with an accuracy

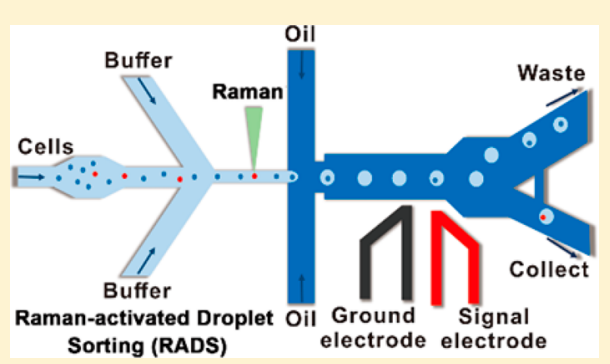
of $98.3 \%$, an enrichment ratio of eight folds, and a throughput of $\sim 260$ cells $/ \mathrm{min}$. Of the RADS-sorted cells, $92.7 \%$ remained alive and able to proliferate, which is equivalent to the unsorted cells. Thus, the RADS achieves a much higher throughput than existing RACS systems, preserves the vitality of cells, and facilitates seamless coupling with downstream manipulations such as single-cell sequencing and cultivation.

\begin{abstract}
A single cell is the basic unit of function and evolution for cellular lives on Earth. Thus, accurate and high-throughput sorting of single-cells are key tools for mechanistic dissection of functional heterogeneity among cells and probing yet-to-becultured microbes in nature. ${ }^{1-3}$ A single-cell Raman spectrum (SCRS) provides the intrinsic biochemical profile of a cell at a given state and thus can be considered as a function-based instant portrait-photo of the cell. ${ }^{4}$ Due to its label-free and noninvasive features, Raman-activated cell sorting (RACS), which sorts cells based on their SCRS, has attracted increasing interest.

Recently, a series of core technologies and devices for RACS have emerged. ${ }^{5-7}$ By coupling Raman microspectroscopy with optical tweezers (OT) and laser ejection, respectively, static versions of RACS called Raman tweezers ${ }^{8,9}$ and Ramanactivated cell ejection (RACE) ${ }^{10,11}$ were developed. Although these systems were simple and practical, the relatively low sorting throughput greatly hampers their broader applications. To achieve higher throughput, flow-mode RACS named Raman-activated microfluidic sorting (RAMS) was introduced based on a trap-and-release strategy. ${ }^{12-16}$ Among these optical tweezer based RAMS systems, cells were immobilized for Raman measurement and then targeted cells were dragged to a
\end{abstract}

designated location for collection. ${ }^{12-16}$ However, the very low throughput $\left(\sim 3 \mathrm{~min}\right.$ per cell $\left.{ }^{5}\right)$ of optical tweezers was still an unsolved problem; moreover, photodamage to the target cells can be significant when laser tweezers of visible wavelengths were used. $^{17}$

To tackle these limitations which are inherent to optical tweezers, we have recently developed a high-speed RACS system that combines positive dielectrophoresis ( $\mathrm{pDEP}$ ) for the efficient trap-and-release of cells with a solenoid-valve-suctionbased switch ${ }^{18}$ for cell separation. ${ }^{19}$ Compared to the optical tweezers based RACS, throughput of our RACS system, at approximately one cell per second, represents a significant increase. However, it was difficult to further increase the throughput, due to the response delay of the solenoid and the time delay required to resume stability of the fluids after suction. On the other hand, Mcllvenna et al. proposed a pressure-dividers-based switch to sort the cells with high accuracy, yet the low throughput $(0.5 \mathrm{~Hz}$, i.e., one cell every 2

Received: September 22, 2017

Accepted: November 3, 2017

Published: November 3, 2017 


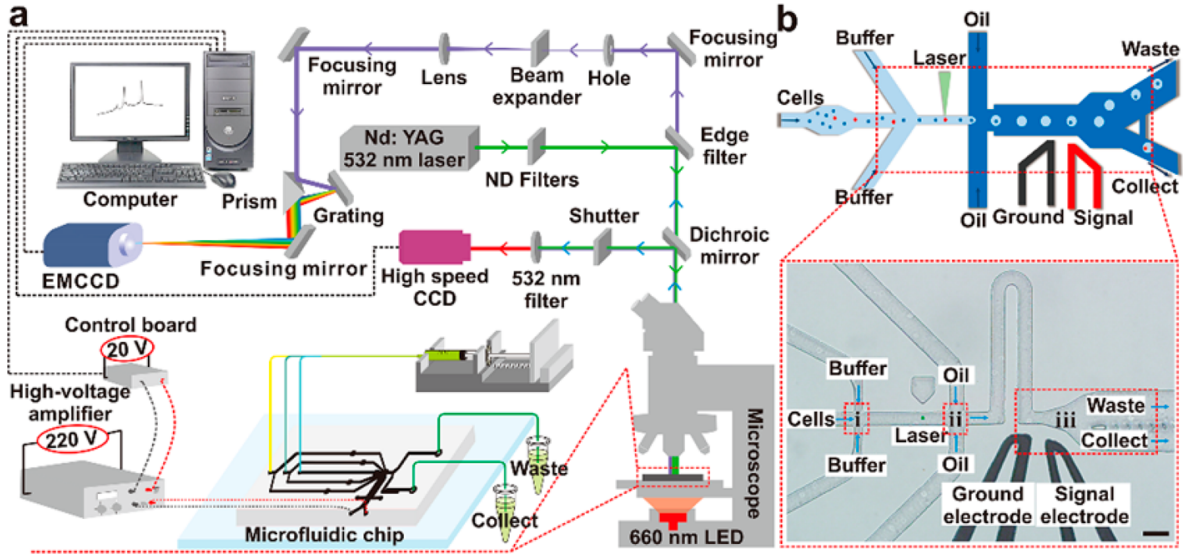

Figure 1. Overview of RADS system setup for high-throughput screening of AXT-hyperproducing H. pluvialis cells. (a) Schematic diagram of RADS system setup. (b) Schematic illustration of the chip design. The red spheres represent target cells, and the green triangle indicates the position of the $532 \mathrm{~nm}$ laser beam. The inserted photograph is a bright-field image of the main functional units: i, pinch flow focusing unit; ii, cross-junction based droplet generation unit; and iii, DEP-based droplet sorting unit. Scale bar represents $100 \mu \mathrm{m}$.

s) was still recognized as one critical issue that remains to be resolved. $^{20}$

Droplet-based microfluidics has shown substantial promises in the past few years. ${ }^{21}$ In fluorescence-activated cell sorting (FACS), coupling compartmentalization of individual cells in microdroplets and DEP-based cell sorting can achieve ultrahigh throughput and moreover can be readily coupled to downstream droplet-based DNA/RNA extraction or cell culture, $^{22-24}$ e.g., isolation of cells in droplets can improve recovery rate of slow-growing species. ${ }^{25}$ However, dropletbased microfluidic RACS prototypes have not been demonstrated, which may be due to several reasons: (i) the convex/ concave shape of the droplet surface creates a lensing effect that distorts the focus and reduces the spatial resolution, making it difficult to accurately acquire the Raman signals of dropletencapsulated cells; (ii) the strong Raman background of oil medium can adversely affect the system's ability to accurately distinguish the Raman signals of droplet-encapsulated cells; and (iii) the difficulty in integrating SCRS acquisition and analysis, cell encapsulation and sorting within an automated system.

In this work, we established a droplet-based microfluidic RACS named Raman-activated droplet sorting (RADS). Key features of the RADS system include: (i) cells were interrogated for SCRS prior to droplet generation, which eliminates the interference from droplet surface and oil, and (ii) single-cell droplet encapsulation was directly coupled to DEP-based droplet sorting, which simplifies the system design and increases sorting throughput and accuracy. Consequently, all single-cells were encapsulated into droplets after Raman detection, while only those droplets that contain the target cells would trigger the DEP after a specific delay and thus be sorted into the collecting channel. Furthermore, a homemade software, QSpec, was developed to automatically integrate and synchronize the RADS system.

As a proof of concept for RADS, we employed microalgal strain selection for astaxanthin (AXT) as a model. AXT is one of the most potent antioxidants known and has found great commercial potential in aquaculture, pharmaceutical, and food industries. AXT is naturally produced by Haematococcus pluvialis (H. pluvialis), ${ }^{26}$ a unicellular freshwater green microalga; thus, the ability to rapidly screen AXT-hyperproducing $H$. pluvialis cells is pivotal to large-scale production of AXT. Traditionally, the screening process is based on cell culture (e.g., plate-based colony cultivation followed by liquid culture), which however has been time-consuming and low-throughput due to the low growth rate and susceptibility to contamination of $\mathrm{H}$. pluvialis cells. ${ }^{27} \mathrm{Via}$ an improved FACS technique for AXT, Ukibe et al. were able to screen AXT-hyperproducing mutants of the yeast Xanthophyllomyces dendrorhous. ${ }^{28}$ However, they failed to screen AXT-hyperproducing $H$. pluvialis cells, because fluorescence intensity of the large amount of chlorophyll in microalgal cells masks that of AXT. In addition, infrared (IR) spectroscopy was used for screening AXThyperproducing $H$. pluvialis cells. ${ }^{29,30}$ However, either AXT extraction from cells with organic solvents or cell drying on $\mathrm{BaF}_{2}$ substrates at $40{ }^{\circ} \mathrm{C}$ was required by $\mathrm{IR}$, which makes the procedure very tedious and in vivo screening of live cells not practical. Unlike IR, Raman spectroscopy is friendly to aqueous samples and thus ideal for analyzing live cells, as water molecules do not exhibit strong Raman scattering features. As a result, Raman spectroscopy has been employed for quantitative profiling distribution, structural change and in vivo kinetics of AXT in $H$. pluvialis cells in a noninvasive and label-free manner. ${ }^{30-34}$

To validate the RADS system, $H$. pluvialis cells containing different levels of AXT were mixed and underwent RADS. Those AXT-hyperproducing cells were sorted with an accuracy of $98.3 \%$, an enrichment ratio of eight folds, and a throughput of $\sim 260$ cells $/ \mathrm{min}$. Of the RADS-sorted cells, $92.7 \%$ remained alive and able to proliferate, which is equivalent to the unsorted cells. Thus, the RADS achieves a much higher throughput than existing RACS systems, preserves the vitality of cells, and facilitates seamless coupling with downstream manipulations such as single-cell sequencing and cultivation.

\section{EXPERIMENTAL SECTION}

Materials and Reagents. Chemicals such as ethanol, isopropanol, and Span 80 were purchased from Sinopharm Chemical Reagent (Shanghai, China). Mineral oil, L-asparagine, yeast extract, propidium iodide (PI), PF 127, and $\mathrm{Na}_{2}$ EDTA. $2 \mathrm{H}_{2} \mathrm{O}$ were purchased form Sigma-Aldrich (St. Louis, MO, U.S.A.). SU-8 (GM 3025) was purchased from MicroChem (Massachusetts, U.S.A.). Polydimethylsiloxane (PDMS Sylgard 184) and curing agent (Sylgard 184) were purchased from Dow Corning (Midland, MI, U.S.A.). All regents used in our experiment were analytical grade unless otherwise stated. All 
solutions were prepared with deionized water and were sterilized by filtration $(0.22 \mu \mathrm{m}$ microporous membrane filtration) or via an autoclave $\left(121^{\circ} \mathrm{C}, 30 \mathrm{~min}\right)$ prior to use.

Cell Culture and Preparation. H. pluvialis (NIES-144) was purchased from the National Institute for Environmental Studies (NIES, Japan). Single colonies from basal medium plates $^{35}$ were inoculated into a liquid basal medium at $22{ }^{\circ} \mathrm{C}$ under continuous low light illumination $\left(20 \mu \mathrm{mol}\right.$ photons $\mathrm{m}^{-2}$ $\mathrm{s}^{-1}$ ) with shaking manually once per day. To induce AXT accumulation, exponentially growing cells were resuspended into modified BBM medium (without nitrogen source and containing $10 \mathrm{mM}$ sodium acetate) in triplicate and exposed to continuous illumination of $150 \mu \mathrm{mol}$ photons $\mathrm{m}^{-2} \mathrm{~s}^{-1}$ for various days (to generate a gradient of averaged AXT content in the cells). Batches of cells, with various average AXT contents, were harvested and filtered with a cell strainer ( $40 \mu \mathrm{m}$ microporous membrane filtration) to remove debris and cell clusters and then were washed with sterile deionized water under $1000 \mathrm{~g}$. Cell concentration was measured using a cell count plate and was adjusted to $\sim 8.02 \times 10^{6}$ cells $\mathrm{mL}^{-1}$. Then cells from batches with different averaged AXT contents were mixed to achieve a specific cell ratio. The actual cell ratio was later determined by sampling $>100$ cells using the Raman Points Mapping mode.

Chip Design and Fabrication. We designed a microfluidic chip with PDMS as the structural material. The channel geometry (schematic illustrated in Figure 1a,b) was designed using AutoCAD 2013 (Autodesk, U.S.A.). The width of the channels for loading the cells was designed as $50 \mu \mathrm{m}$. The microfluidic chip was fabricated via soft lithography and rapid prototyping techniques. ${ }^{36}$ Briefly, a SU-8 mold with $50 \mu \mathrm{m}$ in height was fabricated on a silicon wafer. The PDMS layer was produced by pouring a mixture of PDMS and curing agent in a mass ratio of $10: 1$ onto the SU- 8 mold. After curing at $70{ }^{\circ} \mathrm{C}$ for $2 \mathrm{~h}$ in an oven, the PDMS sheet with channel networks was cut and peeled off from the mold. The holes of inlets and outlets were punched using a $0.75 \mathrm{~mm}$-diameter Harris UniCore biopsy punch (Electron Microscopy Sciences). After oxygen plasma treatment (PLASMA-PREEN II-862, Plasmatic Systems, Inc., United States), the PDMS sheet was bonded with a PDMS-coated glass substrate. After fabrication, the device was heated to $100^{\circ} \mathrm{C}$ on the hot plate, and a low melting point In-Sn solder was filled into the electrode channels. Small pieces of copper wire were inserted to make the electrical connection with the solder electrodes.

System Setup. System setup is shown in Figure 1a. The PEEK tubing (O.D. $=0.03$ in., I.D. $=0.012$ in.; Cole-Parmer, U.S.A.) was used to connect the microfluidic device, syringe equipped on the pumps (LSP01-2A, Longer Pumps, China), and tube for cell collection. The tubing and syringe for cell loading were treated with hydrophilic regent " $5 \%$ PF 127 " for 10 $\mathrm{min}$ and washed with sterile deionized water for $1 \mathrm{~min}$ prior to operation. Mineral oil containing $2.5 \%(\mathrm{w} / \mathrm{w})$ Span 80 surfactant was used for droplet generation. A high-voltage amplifier (PC 2000, Tianjin Dongwen High Voltage Power Supply Co. Ltd., China), controlled by a digital I/O unit (DIO1616LX-USB, CONTEC) connected with the computer, was employed to generate DEP to trigger the target droplet sorting.

Raman microscopy was carried out on a customized LabRam HR micro-Raman setup, ${ }^{10,19}$ which is equipped with a Nd:YAG $532 \mathrm{~nm}$ laser emitter (Ventus, Laser Quantum Ltd., United Kingdom) as the excitation light source, an electron-multiplying charge-coupled device (EMCCD; Newton DU970N-BV,
Andor, United Kingdom) for collecting the Raman signals, a high-speed CCD camera (Pike F-032, Allied Vision Technologies, China) for monitoring the cell and droplet flow, and a $60 \times$ water objective $(\mathrm{NA}=1.0$, Olympus, United Kingdom $)$ to focus the laser beam on the sample. A 600 lines $/ \mathrm{mm}$ grating was used for the measurements, resulting in a spectral resolution of $\sim 1 \mathrm{~cm}^{-1}$ with 1581 data points ranging from 600 to $2100 \mathrm{~cm}^{-1}$. A $660 \mathrm{~nm} \mathrm{LED} \mathrm{array} \mathrm{was} \mathrm{used} \mathrm{as} \mathrm{the} \mathrm{light}$ source for monitoring the sorting process.

A homemade software, QSpec, was developed to control the electronics (EMCCD and high-voltage amplifier, etc.) and adjust the system parameters (e.g., acquisition time and DEP duration). With the QSpec, all of the RADS units including microfluidic device, Raman system, and DEP system were integrated and can be operated in an automatic mode.

Operation Procedure for RADS. There are five steps for the cell sorting, as shown in Figure 2 and Video S1 in the

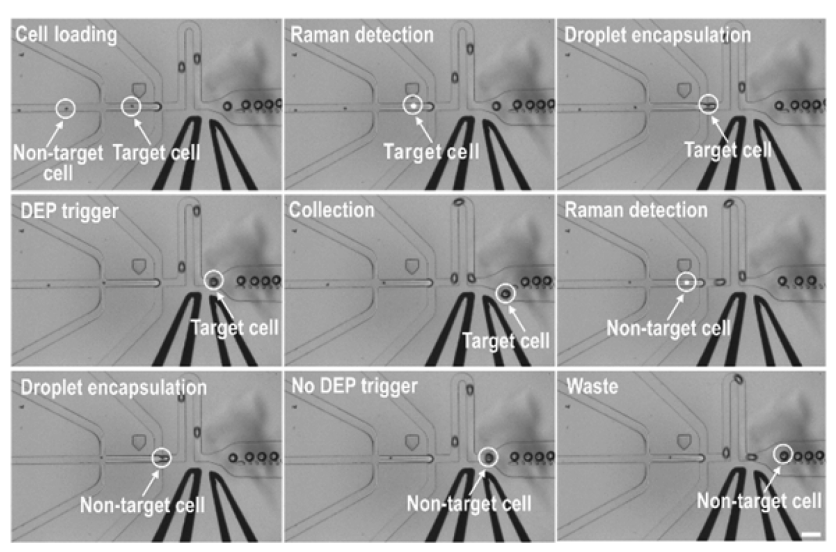

Figure 2. Step-by-step operation process for DEP-based single-cell sorting. (1-5) Trajectory of the target cell, consequently sorted into the collecting channel based on the DEP trigger. (6-9) Trajectory of the nontarget cell, resulting in flowing into the waste channel without the DEP trigger. Scale bar represents $200 \mu \mathrm{m}$.

Supporting Information: Step 1, cell suspension was loaded into the chip; step 2, cells were focused into single-cell flow by two pinch flow; step 3, cells were passed through the laser point and analyzed by Raman; step 4, single-cells were encapsulated into single-droplets; step 5, after a specific delay, target droplets were sorted by DEP and flowed into collecting channel, while nontarget droplets would flow into waste channel.

Cell Recovery. The sorted droplets containing target cells were collected and filtered using Parylene C film featuring pores with $12 \mu \mathrm{m}$ in diameter (smaller than the cell size which is $>20 \mu \mathrm{m}$ in diameter). During the filtering, oil would pass through the film easily with a filter paper placed underneath the film, while the cells would be trapped on the film. After washing with culture media, the Parylene C film containing target cells was transferred into the flask containing $15 \mathrm{~mL}$ of basal medium and shaken gently to rinse the cells off from the film. Finally, the Parylene $\mathrm{C}$ film was removed, and the cells were cultured as described above.

Validation and Evaluation Assays. For the assay that validates sorting accuracy, the cells were reinjected into the chip, and the SCRS was acquired without sorting and analyzed to verify whether the sorting result met the sorting criteria. Two assays were employed to evaluate the vitality of postsorting cells. (i) To determine whether the postsorting cells are alive or dead, the cells were stained with PI $\left(5 \mu \mathrm{mol} \mathrm{L}^{-1}\right)$ for $10 \mathrm{~min}$ to 

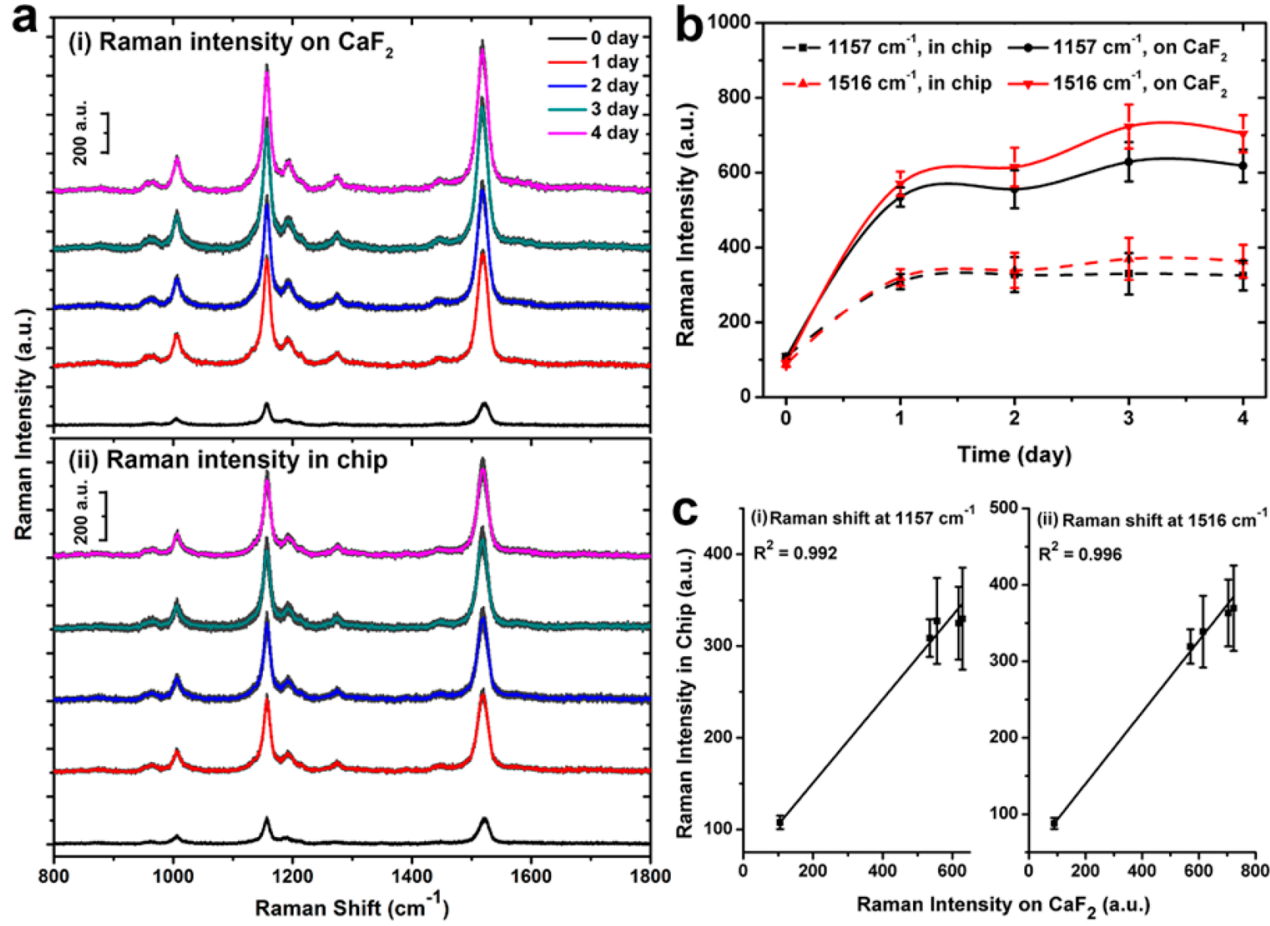

Figure 3. Quantitative Raman detection of AXT in live individual $H$. pluvialis cells. (a) Single-cell Raman spectra of $H$. pluvialis cells induced for different days on $\mathrm{CaF}_{2}$ (i) and in chip (ii). Colored lines and dark gray shading indicate the mean value and the SEM of 60 cells, respectively. (b) Raman intensity of $H$. pluvialis cells induced for different days at 1157 and $1516 \mathrm{~cm}^{-1}$ on $\mathrm{CaF}_{2}$ and in chip. Error bars represent the $S E M$ of 60 cells. (c) Linear correlation between the Raman intensity detected on $\mathrm{CaF}_{2}$ and in chip at $1157 \mathrm{~cm}^{-1}\left(\mathrm{i}, R^{2}=0.992\right)$ and $1516 \mathrm{~cm}^{-1}\left(\mathrm{ii}, R^{2}=0.996\right)$. Error bars represent the SEM of 60 cells.

measure the survival rate. (ii) To assess the robustness of cellular proliferation, the recovered cells were cultured in 15 $\mathrm{mL}$ of basal medium, and the concentration was monitored for 18 days using cell counting plate. For these two assays, the sorting criteria was set as $1516-1800 \mathrm{~cm}^{-1}>200$ (the chip background is $<60)$ to sort the cells, and the unsorted cells were used as the control group.

\section{RESULTS AND DISCUSSION}

RADS Chip Design and System Development. In the present implementation of FADS, the fluorescence signal was measured after single-cell droplet encapsulation. ${ }^{22-24}$ However, owing to the lensing effect of the convex/concave shape of the droplet surface and the strong inherent Raman interference from oil, it was difficult to accurately acquire the SCRS of cells encapsulated in droplets. To tackle this challenge we proposed to interrogate SCRS of the cells prior to droplet encapsulation. Accordingly, we fabricated a RADS (Figure 1b), in which the droplet generation unit (ii) designed to encapsulate single-cells was set after the pinch flow focusing unit (i) that converges cells into the center line of the channel for efficient SCRS acquisition. Based on this design, the efficiency of SCRS acquisition was significantly improved and the effect of droplet on SCRS acquisition completely eliminated. Moreover, the DEP-based droplet sorting unit (iii) designed to isolate the droplets containing the target cells was set directly after the droplet encapsulation unit, so as to realize simultaneous droplet encapsulation and sorting, which increases sorting accuracy and simplifies system design. To bias all droplets flow into the "waste" channel in the absence of DEP, which was a central prerequisite for accurate sorting, resistance of the "collect" channel was designed to be 1.5 times higher than that of the "waste" channel. Furthermore, to balance the resistance changes caused by the bias of droplet flow, the sorting junction was implemented as several small islets. These designs endow the RADS chip the ability to screen AXT-hyperproducing $H$. pluvialis cells with high-throughput and high-accuracy.

In our Raman system, the shortest acquisition time for AXT is $\sim 30 \mathrm{~ms}$ under laser power of $100 \mathrm{~mW}$ with 1000 -fold degeneration (the laser power on sample is $\sim 135 \mu \mathrm{W}$; Figure S1a). Based on the fact that the cell size used in our experiments is $\sim 30 \mu \mathrm{m}$ in diameter, the optimized cell velocity in the channel should be $1 \mathrm{~mm} \mathrm{~s}^{-1}$. Based on the relationship between flow velocity and cell velocity (Figure S1b), the flow velocity for loading the cells should be $7 \mu \mathrm{L} \mathrm{h}^{-1}\left(4 \mu \mathrm{L} \mathrm{h}^{-1}\right.$ for cell sample and $3 \mu \mathrm{L} \mathrm{h}^{-1}$ for pinch flow). Under these conditions, the efficiency of SCRS acquisition was increased significantly, to $\sim 98 \%$ (Figure S2 and Video S2).

In the RADS system, DEP-based droplet sorting was applied to isolate those containing target droplets cells. As previously reported, droplet spacing plays an important role in successful sorting. ${ }^{22,37}$ When being physically too close to each other, the droplets would congest at the sorting junction, causing them to flow into the collecting channel in the absence of DEP, and might also induce droplets coalescence in the present of DEP. Increasing the flow rate of mineral oil would increase the droplet spacing. However, the extremely high flow rate of the mineral oil would also decrease the droplet size and increase the droplet speed, which was adverse to efficiency of cell encapsulation and droplet sorting. In our experiments, the optimal flow rate for mineral oil was $120 \mu \mathrm{L} \mathrm{h}^{-1}$, with which the droplets were generated with $50 \mu \mathrm{m}$ in diameter. Under these conditions, application of $3 \mathrm{~ms}$ of an $800 \mathrm{Vp}-\mathrm{p}(-400$ to +400 $\mathrm{V})$ voltage was sufficient for isolation of the target droplets. 

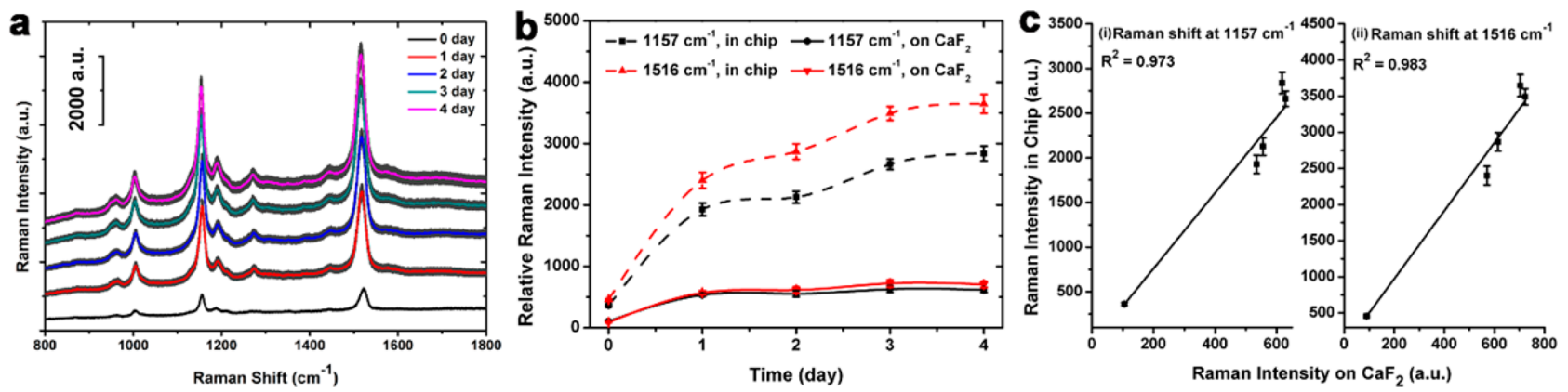

Figure 4. Quantitative characterization of AXT in real-time with raw data in the flow state. (a) Single-cell Raman spectra of $H$. pluvialis cells induced for different days in the RADS chip. Colored lines and dark gray shading indicate the mean value and the SEM of 60 cells, respectively. (b) Relative Raman intensity of $H$. pluvialis cells induced for different days at 1157 and $1516 \mathrm{~cm}^{-1}$ on $\mathrm{CaF}_{2}$ and in chip. Error bars represent the $\mathrm{SEM}$ of 60 cells. (c) The linear correlation between the Raman intensity detected on $\mathrm{CaF}_{2}$ (processed data) and in the $\mathrm{RADS}$ chip (raw data) at $1157 \mathrm{~cm}^{-1}\left(\mathrm{i}, R^{2}=\right.$ 0.973 ) and $1516 \mathrm{~cm}^{-1}$ (ii, $\left.R^{2}=0.983\right)$. Error bars represent the SEM of 60 cells.

To increase the portion of droplets containing only one cell and reduce that of those harboring multiple cells, samples of $\sim 8.02 \times 10^{6}$ cells $\mathrm{mL}^{-1}$ (the final concentration was $\sim 4.58 \times$ $10^{6}$ cells $\mathrm{mL}^{-1}$ due to the loading of pinch flow) were loaded to encapsulate theoretically $\lambda=0.3$ cells in droplets with $\sim 50 \mu \mathrm{m}$ diameter. As estimated by the Poisson statistics, this setting of cell density would result in $74.08 \%$ of the droplets containing no cells, $22.22 \%$ containing a single cell, $3.3 \%$ containing two cells, and $0.38 \%$ containing over two cells. ${ }^{24}$ During sorting, there would be a specific time delay for the flowing cells to reach the sorting junction after SCRS acquisition. Under the above optimized parameters the time delay was calculated to be $145 \mathrm{~ms}$ by recording the real-time cell flow using a high-speed CCD. Hence, the high-voltage amplifier controlled by a digital I/O unit would be triggered to sort the target droplets after 145 ms when the Raman system acquires a signal that meets the sorting criteria. A $660 \mathrm{~nm}$ LED array, which was found to exert no interference on SCRS acquisition, was employed to monitor the entire sorting process in real-time. Finally, to automate the function of each unit and integrate Raman microscopy and DEP-based droplet sorting, the QSpec software was developed.

Quantitative Raman Detection of AXT in the PDMSGlass Hybrid Chip. Previous studies proved that Raman signal can model concentration and distribution of AXT in $H$. pluvialis. ${ }^{30-34}$ The Raman spectrum of AXT showed two most prominent characteristic bands at 1516 and $1157 \mathrm{~cm}^{-1}$ which were assigned to $\mathrm{C}=\mathrm{C}$ and $\mathrm{C}-\mathrm{C}$ stretching vibrations of the chain bonds, respectively. Here, the cells after stress-induced AXT accumulation for various days, which featured distinct averaged AXT concentrations at each of the days, were interrogated for SCRS with laser of $100 \mathrm{~mW}$ and filter of 1000fold degeneration on a calcium fluoride $\left(\mathrm{CaF}_{2}\right)$ slide (Figure 3a (i). These SCRS were then treated as the benchmark curve of AXT concentration in $H$. pluvialis. Our RADS process was carried out on the PDMS-glass hybrid chip. To test whether background interference from PDMS or glass affects SCRSbased measurement of AXT in $H$. pluvialis, the cells were also analyzed in RADS chip and compared to those analyzed on $\mathrm{CaF}_{2}$ slide under identical conditions (Figure 3a (ii). Preprocessing of all of the raw spectra was performed with LabSpec 5 (Horiba Scientific, Orsay, France), including background subtraction and baseline correction via a polynomial algorithm with a degree of seven. As shown in Figure 3a,b, although the Raman intensity measured in the RADS chip decreased significantly at both 1157 and $1516 \mathrm{~cm}^{-1}$ as compared to that measured on the $\mathrm{CaF}_{2}$ slide, the tendency of AXT accumulation over time was identical between RADS chip and $\mathrm{CaF}_{2}$ slide. Furthermore, a good linear relationship between the Raman intensity obtained in RADS chip and on $\mathrm{CaF}_{2}$ slide at both 1157 and $1516 \mathrm{~cm}^{-1}$ was found, as illustrated in Figure 3c. Collectively these data indicated that the Raman background interference from the RADS chip would not compromise the quantitative detection of AXT in H. pluvialis.

Quantitative Characterization of AXT in Real-Time with Raw Data in Flow State. During real-time sorting, the Raman signal was acquired in flow state without preprocessing. Thus, to ensure that such raw data can quantitatively detect AXT, the cells induced for different days were further interrogated for SCRS in flow state. To increase signal intensity in RADS chip, laser of $100 \mathrm{~mW}$ and filter of 100-fold degeneration were applied for SCRS acquisition. The raw spectrum illustrated in Figure $4 \mathrm{a}$ revealed that the Raman intensity of the two characteristic bands at 1516 and $1157 \mathrm{~cm}^{-1}$ was still positively correlated with AXT concentration. The Raman intensity of 1157 and $1516 \mathrm{~cm}^{-1}$ was further characterized using the absolute Raman intensity (defined as the actually detected value) shown in Figure S3a and the relative Raman intensity (defined as the intensity $1157 \mathrm{~cm}^{-1}-$ intensity ${ }_{1800 \mathrm{~cm}^{-1}}$ or intensity ${ }_{1516 \mathrm{~cm}^{-1}}-$ intensity $_{1800 \mathrm{~cm}^{-1}} ; 1800 \mathrm{~cm}^{-1}$ was used due to the absence of biological molecular vibrations from 1800 to $1840 \mathrm{~cm}^{-110}$ ) shown in Figure $4 \mathrm{~b}$, indicating an identical tendency compared with those measured on $\mathrm{CaF}_{2}$ slide. As shown in Figures S3b and 4c, both the absolute and relative Raman intensity exhibited a good liner relationship $\left(R^{2}\right.$ $>0.9$ ) with the benchmark intensity acquired on $\mathrm{CaF}_{2}$ slide, indicating that the raw spectrum acquired in the flow state can also be employed for quantitative characterization of AXT. In fact, compared with the absolute intensity $\left(R^{2}<0.97\right)$, the relative Raman intensity $\left(R^{2}>0.97\right)$ exhibited an even higher, i.e., nearly linear, correlation with the Raman intensity on $\mathrm{CaF}_{2}$ slide. Based on these results, the relative Raman intensity at $1516 \mathrm{~cm}^{-1}$ was chosen as the sorting criterion in the following sorting experiments.

Integration for Automated and Programmable Cell Sorting. To achieve automated and reliable operation of the RADS system with high-throughput and high-accuracy, SCRS acquisition and DEP-based droplet sorting were synchronized via software. The work flow of the whole sorting process is shown in Figure S4. Basically, except the loading of cells, pinch buffer and mineral oil, which was controlled by the pump, the whole process was run automatically based on predefined operational parameters, and the sorting criteria can be set freely 
according to the specific sorting requirement. During the sorting, the Raman spectrum was acquired continuously, resulting in enhancement of spectra analysis speed and sorting throughput. Furthermore, the Raman spectra acquired in realtime were saved automatically, so as to facilitate off-line spectra analysis. It should be noted that the SCRS acquisition and DEP-based droplet sorting were controlled by multithreading, i.e., SCRS acquisition ran simultaneously during the droplet sorting. This design further increased the sorting efficiency.

Validating the Sorting Efficiency of RADS. Traditionally, centrifugation or demulsification reagents such as PicoBreak were used to recover the cells from sorted droplets. ${ }^{38,39}$ These recovery strategies can suffer from drawbacks such as harmful effects on cell and low efficiency. In our experiments, an ultrahigh porosity Parylene $\mathrm{C}$ film featuring pores with 12 $\mu \mathrm{m}$ in diameter (Figure 5a) was used to recover the sorted cells.

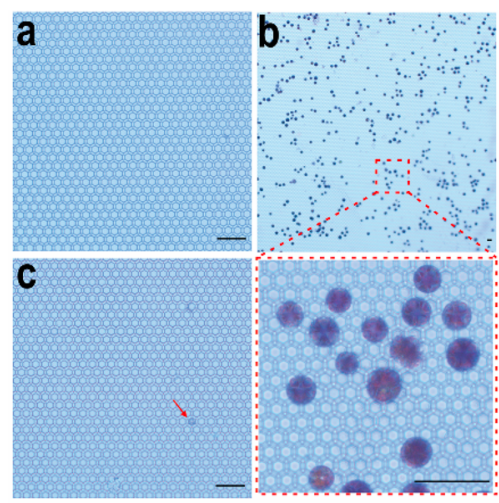

Figure 5. Cell recovery via an ultrahigh porosity Parylene $\mathrm{C}$ film. The images demonstrate the ultrahigh porosity Parylene C film before (a), during (b), and after (c) filtration. The red arrow in panel $c$ indicates the cell attached on the film after washing. Scale bars represent $50 \mu \mathrm{m}$.

The fabrication procedure of the Parylene $\mathrm{C}$ film was described in our previous works. ${ }^{40,41}$ As shown in Figure $5 b$, the cells were trapped on the film with high efficiency and could also be released easily with almost no cells attached on the film after washing (Figure 5c). With this recovery strategy, a high targeted cell separation yield of $\sim 96 \%$ was achieved. ${ }^{40,41}$

To validate the sorting efficiency, cells induced for $0 \mathrm{~d}$ and 3 $\mathrm{d}$ respectively (i.e., in the absence or presence of AXT; Figure 4) were mixed at specific ratios and underwent the RADS, where the sorting criterion was set as $1516-1800 \mathrm{~cm}^{-1}>1200$. As shown in Figure 6a,b, among the over 60 post-RADS cells that were randomly selected and then verified for AXT content, only one cell failed to meet the preset sorting criterion, as indicated by blue spectra. On average, as shown in Figure 6c, the ratio of AXT-hyperproducing cells was elevated to $98 \%$, (from original samples of $12 \%$; three independent experiments), representing around eight folds enrichment.

Furthermore, to test the performance of our RADS system under a more demanding situation, the cells induced for 1 and $2 \mathrm{~d}$ respectively (i.e., the two kinds of cells to be separated both produce AXT, but at different levels of abundance; Figure 4) were mixed at different ratios and then served as starting material for RADS. Correspondingly, the sorting criterion was set as $1516-1800 \mathrm{~cm}^{-1}>2500$. As shown in Figure 6d,e, among the over 60 post-RADS cells randomly selected and verified, only 15 cells failed to meet the sorting criteria, as indicated by blue spectra. On average, as demonstrated in Figure 6f, the ratio of AXT-hyperproducing cells was elevated to 78\%, (from original samples of $11 \%$, three independent experiments), which represented around seven folds enrichment. The sorting efficiency is not $100 \%$, which might be due to several reasons: (i) there was a certain degree of system error in the device setup; (ii) cells were detected in their flow state yet the raw data from the slide were used for setting sorting criteria; (iii) distribution of AXT in $H$. pluvialis cells was not absolutely uniform; and (iv) according to Poisson statistics, there is a very low ratio of droplets containing over one cell.

Theoretically, the sorting throughput under our experimental settings can reach $>500$ cells $\mathrm{min}^{-1}$, which is mainly determined
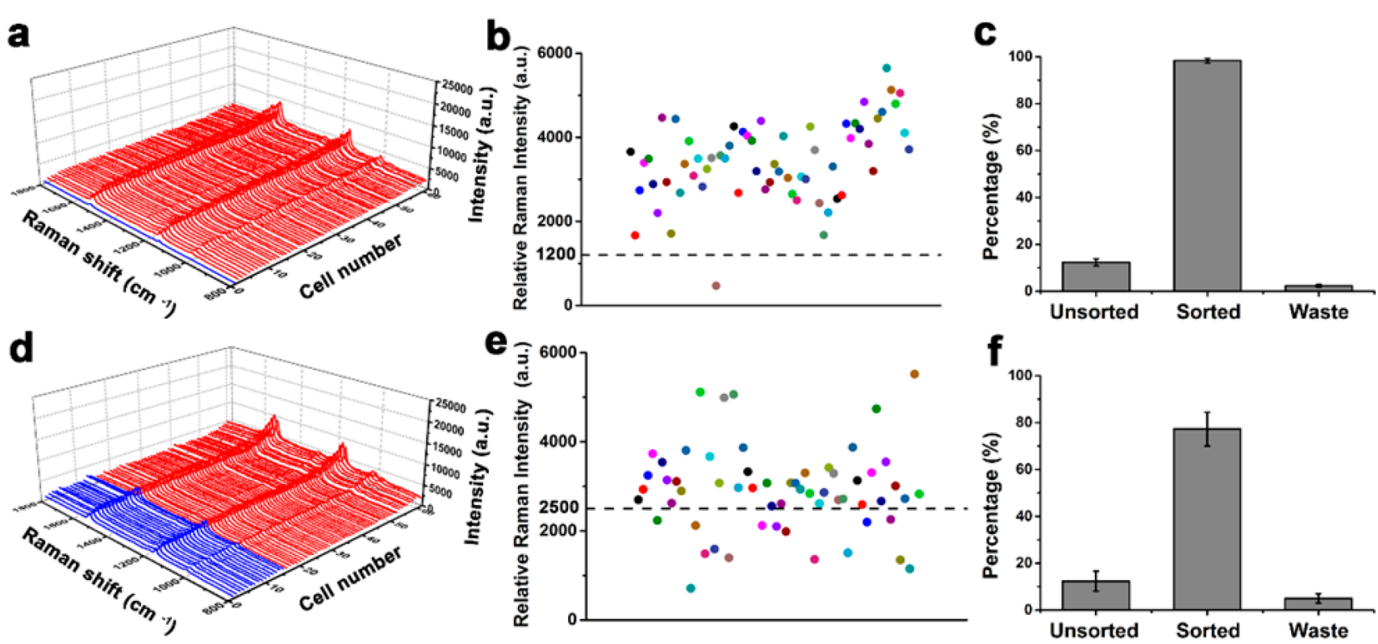

Figure 6. Validation of sorting efficiency of RADS. $(a-c)$ RADS were performed by sorting AXT-hyperproducing cells from a mixture that contained $\sim 12 \%$ target cells (i.e., cells induced for $3 \mathrm{~d}$, which produced AXT), which was generated by mixing the cells induced for $0 \mathrm{~d}$ and those induced for 3 $\mathrm{d}$. (d and e) RADS were performed by sorting AXT-hyperproducing cells from a mixture that contained $\sim 11 \%$ target cells, which was generated by mixing the cells induced for $1 \mathrm{~d}$ and those induced for $2 \mathrm{~d}$. (a and d) Raman spectra of $>60$ sorted cells. Red spectra indicate the target cells, and the blue spectra represent the nontarget cells. ( $b$ and e) Distribution of the relative Raman intensity of sorted cells at $1516 \mathrm{~cm}^{-1}$. The dotted line at 1200 in panel b and at 2500 in panel e indicate the sorting criteria. (c and f) Enrichment of the target cells by the RADS system. "Sorted" indicates the positively sorted cells and "Waste" indicates the negatively sorted cells. Error bars indicate the SEM of three independent experiments. 
by the speed of Raman signal acquisition and single-cell encapsulation. In comparison, our actual sorting throughput is $\sim 260$ cells $\mathrm{min}^{-1}$. The lowered performance is mainly due to the sedimentation of the $H$. pluvialis cells (due to their averaged large size of $\sim 30 \mu \mathrm{m}$ in diameter), which however can be further increased by improving the efficiency of cell loading. Due to the much weaker signal of spontaneous SCRS than that of fluorescence probes and the resulting much longer SCRS acquisition time, throughput of our RADS system is still much lower than present droplet-based FACS systems (hundreds to thousands cells per seconds). ${ }^{22-24}$ However, our RADS system is nonlabeling required and features the highest sorting throughput among existing RACS techniques, such as Raman tweezers (minutes per cell), ${ }^{8,9}$ RACE (minutes per cell), ${ }^{10,11}$ optical tweezer based RAMS (minutes per cell), ${ }^{12-16}$ trap-free RAMS ( $\sim 30$ cells per minute) ${ }^{20}$ and DEP based RAMS $(\sim 60$ cells per minute). ${ }^{19}$ Collectively, these results suggest robust performance of the RADS system in screening AXT-hyperproducing $H$. pluvialis cells.

Assessing the Effect of RADS on Cell Vitality. Considering that cells experienced laser exposure and DEPbased sorting during the screening process, which might introduce a negative effect on activity or vitality of the sorted cells, assays for survival rate and proliferation were conducted. Few dead cells were present, as indicated by PI staining, in both unsorted cells (Figure 7a) and sorted cells (Figure 7b), and no

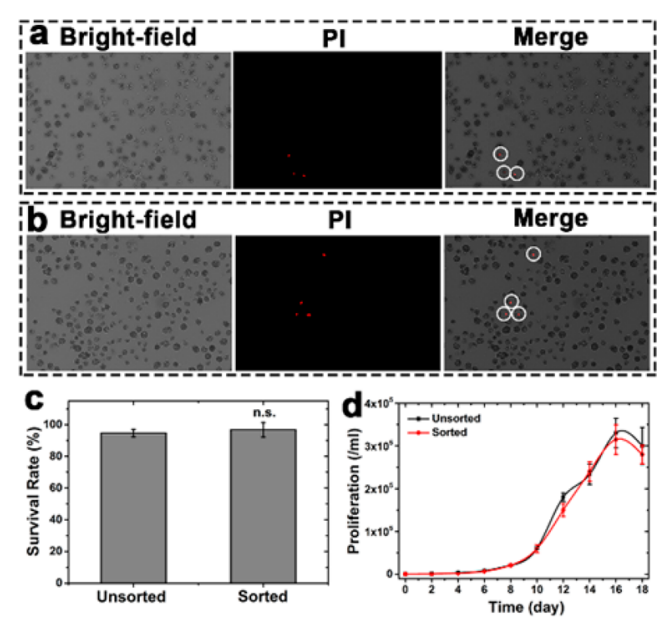

Figure 7. Evaluation of the viability of post-RADS cells. ( $a$ and b) PI staining for detecting dead cells of the control group (a) and the experimental group (b). White circles indicate the dead cells. (c) Survival rate. n.s.: not significant. (d) Proliferation assay. Error bars indicate the SEM of three independent experiments.

significant difference was observed in averaged survival rate (91.9\% for the unsorted cells and $93.7 \%$ for the sorted cells; Figure 7c) between them. Moreover, cellular proliferation was monitored by detecting cell concentration for every $2 \mathrm{~d}$. As shown in Figure $7 d$, the proliferation rate of the sorted cells was nearly identical to that of the unsorted cells, at least within $18 \mathrm{~d}$ post-RADS. The high survival rate of RADS sorted cells was due to several potential reasons: (i) the laser power on the sample was far lower than $1 \mathrm{~mW}$; (ii) the SCRS acquisition time was quite short (30 ms); (iii) SCRS acquisition was carried out in flow state in liquid; (iv) all of the single-cells were encapsulated into droplets, which protect the cells during DEPbased sorting; and (v) Parylene C film was used to recover the sorted cells, which was gentle and harmless to the cells.
Collectively, all these noninvasive procedures have resulted in the high survival rate of RADS-sorted cells.

\section{CONCLUSIONS}

In this study, we established a RADS system that automatically integrates SCRS acquisition and DEP-based droplet sorting in a mode of continuous flow. In our RADS, the droplet technique was adopted to encapsulate and sort single-cells, which combines many of the advantages of plate screening and existing RACS systems: (i) dielectrophoresis (for sorting), which can be difficult to apply directly to cells in suspension, can be used to sort droplets with good stability and high throughput; (ii) vitality of cells can be protected during sorting, i.e., recovery rate of viable cells postsorting is improved; (iii) sorted cells are still compartmentalized in droplets, which facilitates seamless integration with downstream extraction of DNA, RNA, proteins, and metabolites from the cells for further analysis (e.g., sequencing); and (iv) coupling with high sensitivity Raman techniques (e.g., coherent anti-Stokes Raman spectroscopy and stimulated Raman scattering) is facilitated, which might lead to ultrahigh throughput RADS system. By acquiring Raman signals of individual cells prior to droplet encapsulation, the system successfully tackles the difficulties associated with Raman interrogation of dropletencapsulated cells: primarily the lensing effect of the convex/ concave shape of the droplet surface and the strong inherent Raman interference from oil medium. Moreover, droplet encapsulation was directly coupled to DEP-based droplet sorting, which simplifies system design and operation. Finally, based on our RADS system, AXT-hyperproducing $H$. pluvialis cells were sorted with high-throughput $\left(\sim 260\right.$ cells $\left.\mathrm{min}^{-1}\right)$ and high-accuracy ( 98\%), achieving enrichment ratio of eight folds on average.

Our recent work suggested that SCRS, via the ramanome approach, can quantitatively distinguish bacterial species, ${ }^{10}$ measure the general metabolic activity of cells, or probe the catabolic activity targeting a specific substrate, ${ }^{9}$ model the intracellular levels of triacylglycerols (TAG) ${ }^{42}$ and starch, ${ }^{43}$ measure drug sensitivity, ${ }^{44}$ distinguish cellular drug responses based on cytotoxicity mechanism ${ }^{45}$ and trace cross-species metabolite exchange. ${ }^{46}$ Hence, by coupling with the ramanome approach, the RADS can enable the sorting for a broad range of (and theoretically unlimited) cellular functions. On the other hand, the "trap-free" SCRS acquisition strategy adopted in this RADS system might have certain limitations, for example, (i) longer SCRS acquisition time might be required, e.g., when the signal associated with the targeted phenotype in an SCRS is weak, and (ii) small-size cells such as E. coli might require additional optimization, due to the reduced Raman scattering cross-section. As a result, the RADS at its current form is the most suitable for sorting cells that feature high Raman intensity and large scattering cross-section. To tackle these limitations, one potential solution is a RADS chip in which pDEP-based single-cell trap-and-release for SCRS acquisition was performed before droplet encapsulation and sorting. As pDEP-based single-cell trap-and-release has been established as a generic strategy to interrogate the intrinsic SCRS of cells, ${ }^{19}$ the pDEPRADS should be feasible and can tackle those cells with weak SCRS signal, by extending the exposure time of cells during SCRS acquisition. We envision that RADS may become a generally applicable, versatile, and high-throughput RACS platform for label-free functional screening of cells and subsequent omics profiling at single-cell resolution. 


\section{ASSOCIATED CONTENT}

\section{S Supporting Information}

The Supporting Information is available free of charge on the ACS Publications website at DOI: 10.1021/acs.analchem.7b03884.

Optimization of the Raman acquisition time and flow velocity (Figure S1); time-lapse images showing the process of single cell passing the laser and the continuous delivery of single cells to the laser point (Figure S2); quantitative characterization of AXT in real-time with raw data in flow state (Figure S3); and workflow of the QSpec software for RADS (Figure S4). (PDF)

Operation concept of RADS-based screening of AXThyperproducing cells. (AVI)

Highly efficient Raman detection of the cells in flow. (AVI)

\section{AUTHOR INFORMATION}

\section{Corresponding Authors}

*E-mail: xujian@qibebt.ac.cn. Phone: +86-0532-80662653.

*E-mail: mabo@qibebt.ac.cn. Phone: +86-0532-80662657.

ORCID

Xixian Wang: 0000-0003-4856-6516

Jian Xu: 0000-0002-8380-6637

\section{Author Contributions}

${ }^{\perp}$ X.W. and L.R. contributed equally.

\section{Notes}

The authors declare no competing financial interest.

\section{ACKNOWLEDGMENTS}

Project funded by China Postdoctoral Science Foundation. The authors gratefully acknowledge financial support from National Natural Science Foundation of China (31600076, 31470220, 31670101, 31327001, 31601084, 21775155, and 31425002), Ministry of Science and Technology of China (2016YFF0202303), Chinese Academy of Sciences (KFJ-SWSTS-165 and XDBP0305), China Postdoctoral Science Foundation Grant (2017M612364 and 2017T100522), and Shandong Province Natural Science Foundation (ZR2014CQ005). We thank the partial support from State Development \& Investment Corporation (SDIC).

\section{REFERENCES}

(1) Navin, N.; Kendall, J.; Troge, J.; Andrews, P.; Rodgers, L.; McIndoo, J.; Cook, K.; Stepansky, A.; Levy, D.; Esposito, D.; Muthuswamy, L.; Krasnitz, A.; McCombie, W. R.; Hicks, J.; Wigler, M. Nature 2011, 472, 90-94.

(2) Rinke, C.; Schwientek, P.; Sczyrba, A.; Ivanova, N. N.; Anderson, I. J.; Cheng, J. F.; Darling, A.; Malfatti, S.; Swan, B. K.; Gies, E. A.; Dodsworth, J. A.; Hedlund, B. P.; Tsiamis, G.; Sievert, S. M.; Liu, W. T.; Eisen, J. A.; Hallam, S. J.; Kyrpides, N. C.; Stepanauskas, R.; Rubin, E. M.; Hugenholtz, P.; Woyke, T. Nature 2013, 499, 431-437.

(3) Rosenfeld, N.; Young, J. W.; Alon, U.; Swain, P. S.; Elowitz, M. B. Science 2005, 307, 1962-1965.

(4) Xu, J.; Ma, B.; Su, X. Q.; Huang, S.; Xu, X.; Zhou, X. D.; Huang, W. E.; Knight, R. Engineering 2017, 3, 66-70.

(5) Song, Y.; Yin, H.; Huang, W. E. Curr. Opin. Chem. Biol. 2016, 33, $1-8$.

(6) Zhang, Q.; Zhang, P.; Gou, H.; Mou, C.; Huang, W. E.; Yang, M.; Xu, J.; Ma, B. Analyst 2015, 140, 6163-6174.

(7) Chrimes, A. F.; Khoshmanesh, K.; Stoddart, P. R.; Mitchell, A.; Kalantar-Zadeh, K. Chem. Soc. Rev. 2013, 42, 5880-5906.
(8) Huang, W. E.; Ward, A. D.; Whiteley, A. S. Environ. Microbiol. Rep. 2009, 1, 44-49.

(9) Berry, D.; Mader, E.; Lee, T. K.; Woebken, D.; Wang, Y.; Zhu, D.; Palatinszky, M.; Schintlmeister, A.; Schmid, M. C.; Hanson, B. T.; Shterzer, N.; Mizrahi, I.; Rauch, I.; Decker, T.; Bocklitz, T.; Popp, J.; Gibson, C. M.; Fowler, P. W.; Huang, W. E.; Wagner, M. Proc. Natl. Acad. Sci. U. S. A. 2015, 112, E194-203.

(10) Wang, Y.; Ji, Y.; Wharfe, E. S.; Meadows, R. S.; March, P.; Goodacre, R.; Xu, J.; Huang, W. E. Anal. Chem. 2013, 85, 1069710701.

(11) Song, Y.; Kaster, A. K.; Vollmers, J.; Song, Y.; Davison, P. A.; Frentrup, M.; Preston, G. M.; Thompson, I. P.; Murrell, J. C.; Yin, H.; Hunter, C. N.; Huang, W. E. Microb. Biotechnol. 2017, 10, 125-137.

(12) Dochow, S.; Krafft, C.; Neugebauer, U.; Bocklitz, T.; Henkel, T.; Mayer, G.; Albert, J.; Popp, J. Lab Chip 2011, 11, 1484-1490.

(13) Dochow, S.; Beleites, C.; Henkel, T.; Mayer, G.; Albert, J.; Clement, J.; Krafft, C.; Popp, J. Anal. Bioanal. Chem. 2013, 405, 27432746.

(14) Lau, A. Y.; Lee, L. P.; Chan, J. W. Lab Chip 2008, 8, 11161120.

(15) Casabella, S.; Scully, P.; Goddard, N.; Gardner, P. Analyst 2016, 141, 689-696.

(16) Xie, C.; Chen, D.; Li, Y. Q. Opt. Lett. 2005, 30, 1800-1802.

(17) Pilat, Z.; Jezek, J.; Sery, M.; Trtilek, M.; Nedbal, L.; Zemanek, P. J. Photochem. Photobiol., B 2013, 121, 27-31.

(18) Zhang, Q.; Zhang, P.; Su, Y.; Mou, C.; Zhou, T.; Yang, M.; Xu, J.; Ma, B. Lab Chip 2014, 14, 4599-4603.

(19) Zhang, P.; Ren, L.; Zhang, X.; Shan, Y.; Wang, Y.; Ji, Y.; Yin, H.; Huang, W. E.; Xu, J.; Ma, B. Anal. Chem. 2015, 87, 2282-2289.

(20) Mcllvenna, D.; Huang, W. E.; Davison, P.; Glidle, A.; Cooper, J.; Yin, H. Lab Chip 2016, 16, 1420-1429.

(21) Shang, L.; Cheng, Y.; Zhao, Y. Chem. Rev. 2017, 117, 79648040.

(22) Baret, J. C.; Miller, O. J.; Taly, V.; Ryckelynck, M.; El-Harrak, A.; Frenz, L.; Rick, C.; Samuels, M. L.; Hutchison, J. B.; Agresti, J. J.; Link, D. R.; Weitz, D. A.; Griffiths, A. D. Lab Chip 2009, 9, 18501858.

(23) Agresti, J. J.; Antipov, E.; Abate, A. R.; Ahn, K.; Rowat, A. C.; Baret, J. C.; Marquez, M.; Klibanov, A. M.; Griffiths, A. D.; Weitz, D. A. Proc. Natl. Acad. Sci. U. S. A. 2010, 107, 4004-4009.

(24) Mazutis, L.; Gilbert, J.; Ung, W. L.; Weitz, D. A.; Griffiths, A. D.; Heyman, J. A. Nat. Protoc. 2013, 8, 870-891.

(25) Liu, W.; Kim, H. J.; Lucchetta, E. M.; Du, W.; Ismagilov, R. F. Lab Chip 2009, 9, 2153-2162.

(26) Boussiba, S. Physiol. Plant. 2000, 108, 111-117.

(27) Sandesh Kamath, B.; Vidhyavathi, R.; Sarada, R.; Ravishankar, G. A. Bioresour. Technol. 2008, 99, 8667-8673.

(28) Ukibe, K.; Katsuragi, T.; Tani, Y.; Takagi, H. FEMS Microbiol. Lett. 2008, 286, 241-248.

(29) Liu, J. H.; Song, L.; Huang, Q. Lett. Appl. Microbiol. 2016, 62, 185-191.

(30) Liu, J.; Huang, Q. Appl. Spectrosc. 2016, 70, 1639-1648.

(31) Kaczor, A.; Turnau, K.; Baranska, M. Analyst 2011, 136, 11091112.

(32) Kaczor, A.; Baranska, M. Anal. Chem. 2011, 83, 7763-7770.

(33) Collins, A. M.; Jones, H. D.; Han, D.; Hu, Q.; Beechem, T. E.; Timlin, J. A. PLoS One 2011, 6, e24302.

(34) Li, K.; Cheng, J.; Ye, Q.; He, Y.; Zhou, J.; Cen, K. Bioresour. Technol. 2017, 244, 1439-1444.

(35) Kobayashi, M.; Kakizono, T.; Nagai, S. J. Ferment. Bioeng. 1991, $71,335-339$.

(36) Duffy, D. C.; McDonald, J. C.; Schueller, O. J.; Whitesides, G. M. Anal. Chem. 1998, 70, 4974-4984.

(37) Fiedler, B. L.; Van Buskirk, S.; Carter, K. P.; Qin, Y.; Carpenter, M. C.; Palmer, A. E.; Jimenez, R. Anal. Chem. 2017, 89, 711-719.

(38) Liu, X.; Painter, R. E.; Enesa, K.; Holmes, D.; Whyte, G.; Garlisi, C. G.; Monsma, F. J.; Rehak, M.; Craig, F. F.; Smith, C. A. Lab Chip 2016, 16, 1636-1643. 
(39) Zhang, Q.; Wang, T.; Zhou, Q.; Zhang, P.; Gong, Y.; Gou, H.; Xu, J.; Ma, B. Sci. Rep. 2017, 7, 41192.

(40) Liu, Y.; Wang, W.; Wu, W.; Li, H.; Sun, Y. MicroTAS 2015, 389-391.

(41) Liu, Y.; Wang, W.; Wu, W.; Li, H.; Yang, F. Transducer 2015, 1767-1769.

(42) Wang, T.; Ji, Y.; Wang, Y.; Jia, J.; Li, J.; Huang, S.; Han, D.; Hu, Q.; Huang, W. E.; Xu, J. Biotechnol. Biofuels 2014, 7, 58.

(43) Ji, Y.; He, Y.; Cui, Y.; Wang, T.; Wang, Y.; Li, Y.; Huang, W. E.; $\mathrm{Xu}$, J. Biotechnol. J. 2014, 9, 1512-1518.

(44) Tao, Y.; Wang, Y.; Huang, S.; Zhu, P.; Huang, W. E.; Ling, J.; $\mathrm{Xu}$, J. Anal. Chem. 2017, 89, 4108-4115.

(45) Teng, L.; Wang, X.; Wang, X.; Gou, H.; Ren, L.; Wang, T.; Wang, Y.; Ji, Y.; Huang, W. E.; Xu, J. Sci. Rep. 2016, 6, 34359.

(46) Wang, Y.; Song, Y.; Tao, Y.; Muhamadali, H.; Goodacre, R.; Zhou, N. Y.; Preston, G. M.; Xu, J.; Huang, W. E. Anal. Chem. 2016, $88,9443-9450$. 